\title{
Consensus et concurrence mémorielles : un enjeu pour les médias
}

Si la transmission orale joue certes encore un rôle aujourd'hui, les études sur la mémoire ne peuvent cependant plus faire l'économie de la dimension médiatique des processus mémoriels, les médias - au sens large - s'étant petit à petit imposés comme supports privilégiés des mémoires collectives. L'ensemble des contributions rassemblées dans ce numéro démontre suffisamment, s'il était besoin, l'importance et le poids des logiques médiatiques à chaque niveau des processus mémoriels; qu'il s'agisse de leurs mécanismes de production, de transmission, de configuration ou de réception. Ainsi les mémoires collectives ne peuvent-elles exister indépendamment des supports médiatiques par lesquels elles s'expriment et accèdent à une existence sociale. Cette remarque invite d'ailleurs à relativiser certaines critiques régulièrement adressées aux médias, dans la mesure où on ne peut concevoir de mémoire sociale "pure», préexistante au processus de médiatisation et que ce dernier viendrait ensuite pervertir ou entacher ; c'est au contraire dans cette dynamique même que se constituent les mémoires collectives.

Comprendre et évaluer les rapports entre médias et mémoires suppose alors d'analyser ces processus dynamiques, dans leurs mouvements de production-circulation-réception. C'est précisément à cette tâche que sont dévolues les recherches présentées ici, témoignant par ailleurs toutes, à des degrés divers, d'une volonté d'articuler ces différents niveaux de processus. Or ce point de vue tend à mettre en évidence le caractère éminemment construit de la mémoire - ou, pour mieux le dire, perpétuellement reconstruit - tant par les médias eux-mêmes que par les divers acteurs mémoriels qui s'en saisissent pour faire aboutir leurs revendications.

Voilà sans doute un des intérêts majeurs de la démarche adoptée dans ce numéro: aborder la mémoire collective sous un angle médiatique permet de la problématiser en éclairant certaines de ses dimensions fondamentales; son caractère construit et processuel, notamment, mais surtout sa dimension éminemment politique (au triple sens évoqué dans l'introduction), que nous aimerions développer brièvement dans ces conclusions. Ce questionnement politique anime en effet l'ensemble des auteurs de ce numéro, et nous paraît constituer un point de fuite 
stimulant pour la recherche sur les rapports entre médias et mémoires. D'autant plus que les formes de ce questionnement révèlent en filigrane un horizon normatif pour les médias en matière de mémoires, qu'il s'agirait de décrire. À partir de là, de cet idéal plus ou moins assumé de médiatisation mémorielle, on pourrait s'interroger sur les modèles qui sous-tendent ces normes, sur leurs contradictions éventuelles ou sur les limites du rôle des médias en matière mémorielle. Nous ne nous aventurerons pas tout à fait jusque là dans le cadre de ces conclusions mais tâcherons toutefois de proposer quelques balises, quelques pistes de réflexion qui pourraient inspirer des recherches futures en ce sens.

Parmi les recherches compilées ici, le premier constat qui s'impose est que celles qui s'attachent, dans la première partie, à analyser les logiques médiatiques de production et de reproduction mémorielles tendent à mettre en évidence les règles qui président à l'élaboration ou à l'imposition de consensus mémoriels. Tandis que les textes réunis dans la seconde partie, consacrés à l'étude de mouvements de transformation, éclairent davantage les mécanismes de conflits ou de concurrences mémorielles. Pourtant cette association ne va pas de soi ; celle-ci se trouve d'ailleurs partiellement remise en cause, par exemple, dans l'article de Christoph Brüll et Catherine Lanneau, placé à dessein à la charnière des deux parties du numéro. De fait, si leur étude met plutôt en évidence la manière dont se perpétue un espace mémoriel, l'objet même de cette perpétuation consiste en un espace divisé, concurrentiel, dont les deux parties constitutives demeurent dans une relative "panne de dialogue ». De cette façon, on pourrait estimer que les médias qu'ils étudient participent à la fois de la reproduction d'un conflit mémoriel et d'un consensus sur ce conflit.

En outre, la mise en évidence des logiques médiatiques de reproduction mémorielle se trouve le plus souvent alliée à une forme de dénonciation de ces logiques, qui relèveraient principalement d'un rapport de forces nécessairement inégal et excluant. Significativement, ces dénonciations visent surtout des médias « dominants " dans leur champ - qu'il s'agisse de cinéma de divertissement, de littérature scientifique ou d'information. À l'inverse, les logiques créatrices de changement, de transformation tendent à être considérées comme ayant une valeur en soi, comme rétablissant l'équilibre «juste » des forces et la pluralité des voix mémorielles. II ne faudrait cependant pas négliger que l'émergence de nouvelles voix, de nouveaux acteurs et de nouveaux " contre-récits » dans l'espace médiatique sont eux-mêmes, nécessairement, le produit d'un rapport de forces. Ainsi les « contre-récits » cesseront-ils sans doute d'être des "contre-récits " dès lors qu'ils accèdent à une visibilité médiatique. L'essentiel résidant sans doute dans le mouvement luimême, c'est-à-dire dans la possibilité d'un renversement des rapports de forces existants. 
Quoi qu'il en soit, nous trouvons dans ce constat un premier élément du cadre normatif qui est censé régler la médiatisation des mémoires : le renouvellement plutôt que la répétition; l'ouverture au conflit plutôt que la perpétuation du consensus.

\section{La (re)production médiatique du consensus mémoriel}

Étonnamment, c'est dans les études sur la production et la reproduction des consensus mémoriels que ressort le mieux le caractère construit de la mémoire. C'est que l'unification - et, symétriquement, les oublis qu'ils supposent apparaît nécessairement socialement inauthentique, ce qui invite d'autant plus à débusquer le pouvoir dans les méandres des processus de mythification à l'origine de ces consensus. II ressort en tout cas de ces travaux que les médias participent de diverses manières à l'élaboration et à la diffusion du consensus mémoriel; que ce soit en tant qu'acteurs, au travers de pratiques et de logiques qui leurs sont propres, ou en tant que ressources mobilisées par des acteurs, auquel cas ils se font le relais de causes qui leur sont externes.

En amont de la production, le média peut se trouver instrumentalisé par des acteurs au service d'un objectif clairement politique (Virginie Cerdeira ; Sébastien Fevry sur la politique mémorielle de Nicolas Sarkozy; et dans une moindre mesure David Copello) de légitimation d'un pouvoir ou d'une décision, par exemple ; le média est alors considéré comme une ressource stratégique. Dans ce cas, le contenu mémoriel véhiculé est également instrumentalisé et donc plus ou moins radicalement "lissé », par divers procédés, de manière à produire du consensus. Ce lissage, ici intentionnel, consiste en un effacement du caractère pluriel, controversé ou polémique de l'élément mémoriel, pouvant aller jusqu'à la censure pure et simple (Virginie Cerdeira). L'oubli corrélatif à ces procédés d'homogénéisation s'apparente alors à du déni, et la mémoire médiatisée se fait prescriptive, visant une réception "programmée " léguée à la postérité.

Parfois cependant, l'instrumentalisation du média et de la mémoire est moins claire, l'intentionnalité plus douteuse, lorsque l'orientation du contenu mémoriel tient davantage à l'engagement politique plus ou moins conscient de l'auteur - ou de l'éditeur - ou à sa proximité avec le pouvoir (David Copello; Christoph Brüll et Catherine Lanneau) ; ce qui devrait nous amener à considérer différents degrés d'instrumentalisation qui vont jusqu'à la malhonnêteté intellectuelle, au déni de la vérité historique. D'un point de vue normatif, il en ressort qu'en matière mémorielle, le média est censé garantir son indépendance par rapport 
au pouvoir - à moins, bien entendu, qu'il ne s'agisse d'un média explicitement dévolu à la communication publique officielle, mais les médias de communication politique n'ont pas fait l'objet de ce numéro.

Au niveau de la production, les biais dénoncés relèvent moins de l'intentionnalité mais tiennent davantage au fonctionnement de la machine médiatique elle-même, ou aux contraintes qui orientent la production et les pratiques d'un média. On retiendra parmi celles-ci d'abord les conditions socio-économiques de production propres au média (Christoph Brüll et Catherine Lanneau); les modes de financement, la langue ou les caractéristiques du public cible, par exemple. Du côté des pratiques, on relèvera notamment les contraintes liées aux conditions de travail et à la matière mémorielle (Marc Vanesse) - comme la disparition des traces, la difficulté de recueillir des témoignages, etc. - au support médiatique et même au genre (Virginie Cerdeira; Marc Vanesse; Sébastien Fevry).

Au-delà de la production, les conditions médiatiques de circulation des discours sur la mémoire expliquent également comment peut se perpétuer puis s'imposer un consensus autour d'une question mémorielle au départ conflictuelle. Christoph Brüll et Catherine Lanneau montrent bien par quels mécanismes se reproduisent, sur un temps relativement long, deux types de récits mémoriels qui demeurent confinés chacun à leur espace médiatique sans parvenir réellement à dialoguer malgré des tentatives de dépassement que l'on pourrait qualifier d'exogamiques. Ce qui pose d'ailleurs la question d'une éventuelle coïncidence entre espaces médiatiques et espaces mémoriels, nous y reviendrons. Cependant, un consensus mémoriel résulte également de rapports de forces entre des réseaux de diffusion par nature inégaux, comme le démontre David Copello (notamment par la mise en évidence de processus de citations auto-référentielles). L'imposition d'un discours mémoriel dans le temps - au détriment de ce qu'il faut alors bien appeler des "contre-discours" - dépend de la force de son réseau de diffusion médiatique : sa massivité, la pérennité de ses supports, mais aussi la position symbolique des médias qui portent ce discours par rapport aux médias qui tiennent un discours concurrent, voué à l'oubli ou, dans le meilleur des cas, à la marginalité.

Que ce soit au niveau de la production ou de la diffusion, les études rassemblées dans cette première partie mettent en évidence des logiques médiatiques dominantes, qui tendraient " naturellement " à l'élaboration, à la reproduction et à l'imposition de mémoires consensuelles, simplifiées, unifiées, lissées voire a-historiques, c'est-à-dire de mythes, condamnant dans le même mouvement les "contre-mémoires " à la marginalité ou au silence. Ainsi, dans cette version, les médias seraient-ils essentiellement voués à la (re)production de 
consensus mémoriels. Cependant, si ces forces de clôture et de reproduction y apparaissent très prégnantes, les médias ne sont pas pour autant tout à fait incapables d'accueillir la nouveauté et la conflictualité; il ne faudrait dès lors pas négliger les forces de transformation: les deux, bien que de manière apparemment inégale, sont à l'œuvre dans les médias.

On pourrait l'envisager assez simplement en considérant le troisième niveau du processus, à savoir celui de la réception, qui préserve en principe les médias de la pure reproduction autoréférentielle du consensus. Jusqu'ici, aux deux premiers niveaux, la réception n'est conçue que comme "programmée ", passive, docile, inscrite dans la production; comme si la production et la diffusion médiatiques de mémoires homogénéisées suffisaient en elles-mêmes à produire de fait un consensus mémoriel dans le social - qui légitimerait en retour le discours mémoriel tenu par les médias. Or la recherche en communication a suffisamment montré que la bien mal nommée "réception " n'est jamais passive et linéaire, mais au contraire active et créative. Sans développer ici la vaste problématique des usages, notons que la question de la réception paraît d'autant plus cruciale que la mémoire est conçue comme une expérience (Sébastien Fevry; Marc Vanesse) qui, " contrairement à l'histoire, [...] se transmet mais ne s'enseigne pas "', ce qui donne toute son importance aux processus d'appropriation, individuels ou collectifs, constituant la «réception» de la mémoire (Sébastien Fevry, notamment).

\section{Transiormations médiatiques et concurrences mémorielles}

Tout consensus mémoriel est nécessairement discriminant, suppose nécessairement l'oubli et le rejet des mémoires concurrentes et, par conséquent, des porteurs de ces mémoires ${ }^{2}$. De sorte qu'un discours médiatique homogénéisant s'expose à des effets de résistance de la part de ces "récepteurs » exclus des récits mémoriels dominants (pour peu, bien entendu, qu'ils soient exposés aux médias concernés). Les médias n'ont en effet pas d'existence absolument autonome; ils s'adressent toujours à un public, et génèrent une "réception » qui peut avoir, en retour, des effets plus ou moins directs sur la production médiatique, et ce, de plusieurs manières: 
$\diamond$ par des phénomènes de feed-back sur le média considéré (du type courrier des lecteurs, par exemple), qui peuvent amener, à terme, à une évolution et un ajustement de son discours mémoriel ;

$\diamond$ par la création ou l'investissement de médias alternatifs, facilités par le développement d'internet et des réseaux sociaux (Sophie Gebeil ; Morane Chavanon);

$\diamond$ par une prise de parole directement au sein du média ou de l'espace médiatique considéré (Jocelyne Chabot et Richard Godin ; Morane Chavanon);

$\diamond$ par une prise de parole publique ou une prise de position politique, auxquelles les médias ne peuvent demeurer imperméables (Jocelyne Chabot et Richard Godin).

Ainsi la diffusion d'une mémoire consensuelle, dans la mesure où elle est excluante, peut-elle générer des tentatives de réappropriation du discours médiatique par des acteurs porteurs de revendications mémorielles, qui visent à faire entendre et reconnaître un contre-discours, quitte à créer, dans un premier temps, leurs propres médias en marge des médias dominants. Ceci signifie, bien entendu, qu'une contre-mémoire ne peut émerger dans l'espace médiatique que portée par des acteurs, plus ou moins organisés politiquement; et, symétriquement, elle nécessite un public, c'est-à-dire qu'elle doit correspondre à une certaine "demande sociale " (Christoph Brüll et Catherine Lanneau). II demeurera toujours des réceptions silencieuses.

S'il ne faudrait pas se montrer trop optimiste en exagérant l'importance de ces forces de transformation, la prise en compte du niveau de la réception permet tout de même de relativiser les logiques médiatiques dominantes et de considérer la manière dont le média peut réintroduire de la conflictualité dans le consensus mémoriel. De fait, la "réception " engendre à son tour des productions - et inversement, toute production médiatique est elle-même le fruit et le signe d'une réception. Ce sont notamment ces productions de la réception qui font l'objet de la seconde partie de «Médias en jeu, enjeux de mémoires ».

Cependant, si les logiques de renouvellement des récits mémoriels apparaissent au travers de ce numéro comme étant la plupart du temps externes aux médias dominants, comme étant plutôt le fait d'acteurs mémoriels ou de médias alternatifs, rien n'interdit cependant d'envisager des mécanismes de transformation internes, inscrites dans les logiques de production médiatiques elles-mêmes. Comme par exemple, dans les médias d'information, l'exigence de nouveauté, la 
recherche du scoop et la volonté de démythification (Christoph Brüll et Catherine Lanneau) qui, même de manière superficielle, peuvent amener le récit mémoriel médiatique à se renouveler; ou encore l'évolution du discours médiatique au gré de la succession des événements dans le temps (Jocelyne Chabot et Richard Godin).

Dans cette seconde partie consacrée aux logiques de transformation, les rapports de force à l'origine de la production mémorielle, qui ne se manifestaient dans la première partie que par le silence ou l'absence de controverse (voir David Copello), se rendent ici visibles en trouvant à s'exprimer sur une scène médiatique. Toute la question est alors de savoir quelle est cette scène, quel est son public, et de quel degré de légitimité elle jouit dans le champ médiatique.

\section{Des irontières mémorielles aux frontières médiatiques}

Au cœur de la problématique des rapports entre médias et mémoires, on retrouve cette question, esquissée plus tôt, d'une correspondance relative entre espaces médiatiques et espaces mémoriels. On pourrait la poser simplement en ces termes : lorsqu'il y a concurrence manifeste entre deux mémoires collectives ${ }^{3}$, cette concurrence se traduit-elle en controverse médiatique, ou demeuret-elle divisée entre deux espaces médiatiques distincts qui coexistent dans une relative ignorance mutuelle ? Autrement dit, existe-t-il une scène possible pour la concurrence mémorielle? La réponse diffère naturellement selon les cas, mais permet de remettre en perspective les résultats des différentes études compilées dans ce numéro et, au passage, de cadrer de manière réaliste l'optimisme souvent associé aux possibilités offertes par les «nouveaux médias".

La recherche de Jocelyne Chabot et Richard Godin sur le génocide des Arméniens dans la presse canadienne offre un bel exemple d'un conflit mémoriel qui, avec le temps, a pu se déployer sur la scène médiatique dominante, aboutissant finalement à une reconnaissance publique de la mémoire génocidaire arménienne. Dans ce cas, les médias s'offrent comme un lieu potentiellement ouvert à la controverse et au dialogue des mémoires, un lieu capable d'intégrer une contre-mémoire à la mémoire collective. En revanche, le cas de l'histoire politique dans les médias belges étudié par Christoph Brüll et

La concurrence mémorielle renvoie à la « compétition complexe et parfois douloureuse entre des groupes sociaux (entre eux ou vis-à-vis d'une autorité) pour défendre et promouvoir le souvenir de certains faits historiques ». GRANDJEAN Geoffrey, "Pluralité des mémoires collectives et dynamique concurrentielle », in Grandjean Geoffrey et Jamin Jérôme, La concurrence mémorielle, Paris, Armand Colin, coll. « Recherches », 2011, p. 13. 
Catherine Lanneau met en évidence deux mémoires distinctes dont les frontières recouvrent assez nettement celles de deux espaces médiatiques qui ne communiquent guère entre eux. Or, le rapport entre les deux réseaux de diffusion médiatique est ici assez équilibré, si bien qu'aucune des deux mémoires communautaires ne semble menacée d'oubli; mais qu'en est-il lorsque ce n'est pas le cas?

Le déséquilibre est manifeste par exemple, dans l'article de Sophie Gebeil, entre les circuits de diffusion des mémoires de l'immigration maghrébine en France et ceux de la mémoire collective nationale dont elles demeurent exclues. Si les mémoires minoritaires trouvent à s'exprimer dans des médias alternatifs (essentiellement numériques), éventuellement créés à cet effet, on peut toutefois craindre que ce réseau de diffusion médiatique ne recoupe guère celui des médias nationaux, et que les mémoires minoritaires ne demeurent condamnées à la marginalité, sans trouver à s'articuler à la mémoire collective nationale. Significativement, Sophie Gebeil rappelle à quel point l'immigration maghrébine en France reste, dans les représentations médiatiques dominantes, une figure radicale et obsédante de l'altérité.

Dès lors, la diffusion de ces mémoires minoritaires risque de rester confinée à un réseau de médias marginaux ou communautaires, précisément, davantage voués à la transmission à l'intérieur du groupe concerné qu'à la visibilité, à la valorisation et à la reconnaissance de leurs mémoires à l'extérieur du groupe - ce qui semble pourtant être l'un des objectifs avoués.

Cette dernière remarque appelle deux questions, que nous nous proposons d'explorer brièvement : la première a trait à la manière dont les médias participent à la légitimation ou à l'illégitimation d'une mémoire; la seconde tient plutôt à leur rôle dans la désarticulation de mémoires collectives.

\section{Les médias comme lieu d'institution de la légitimité mémo- rielle}

La question de la légitimité - c'est-à-dire l'acceptabilité sociale fondée sur des croyances partagées ${ }^{4}$ - semble être au cœur de la problématique des relations entre médias et mémoires; elle taraude en tout cas, sans exception, tous les auteurs réunis dans ce numéro, indépendamment de la diversité de leurs objets et de leurs approches. Il s'en dégage d'abord au moins trois façons, pour les médias, de 
contribuer à la légitimation d'une mémoire : en tant qu'étalon, acteur et instrument de légitimité mémorielle (voir en particulier Morane Chavanon).

En premier lieu, les médias apparaissent comme un étalon de la légitimité d'une mémoire; ils permettraient d'évaluer son authenticité, sa prégnance dans la société, son caractère plus ou moins largement partagé, son importance relative dans l'identité de la communauté. "Miroir social », pour reprendre l'expression de Patrick Charaudeau, les médias reflètent le monde social et l'opinion publique, et sont par là témoins des mémoires collectives qui traversent la société. Dès lors, le degré de visibilité médiatique d'une mémoire atteste de sa prégnance sociale et assoit, dans le même mouvement, la légitimité du groupe porteur de cette mémoire ${ }^{5}$. Inversement, l'invisibilité d'une mémoire, c'est-à-dire son absence de représentation dans l'espace médiatique, signe son inexistence dans l'espace public, et donc son illégitimité. C'est la raison pour laquelle la visibilité médiatique constitue un élément central des revendications mémorielles.

Deuxièmement, les médias sont considérés comme acteurs, co-producteurs de la légitimité des mémoires : miroir, mais miroir déformant, ils auraient le pouvoir de distribuer la valeur sociale via des procédés de hiérarchisation et de valorisation. En ce sens, ils sont un lieu privilégié d'institution de la légitimité mémorielle, ce qui explique autant les tentatives de réappropriation que la volonté de maîtrise du discours médiatique par les acteurs mémoriels.

Troisièmement, la croyance des acteurs dans ce pouvoir d'institution prêté aux médias en font à la fois un enjeu et une ressource stratégique pour les acteurs mémoriels en quête de légitimation. La médiatisation d'une mémoire est en effet perçue comme un gage suffisant de sa légitimité, et tient lieu d'argument dans le cadre de revendications mémorielles. Or le fait même d'utiliser les médias comme une ressource stratégique dans le cadre de luttes mémorielles confirme et renforce de fait leur caractère institutionnalisant; autrement dit la croyance des acteurs dans le pouvoir des médias contribue à renforcer ce pouvoir.

Cependant, encore faut-il voir de quels médias il s'agit. Car, et c'est sans doute là l'essentiel, la légitimité d'une mémoire médiatisée dépend au fond de la légitimité des médias par lesquels elle se diffuse,

${ }^{5}$ À cet égard, il peut être rappelé que la mémoire collective participe à la construction de l'identité d'un groupe. Comme le rappelle Philippe Raxhon, « les opérations de sélection de la mémoire visent [ ] à une efficacité dans le présent, mais aussi dans l'avenir et son légitimées par la quête identitaire au sein d'une collectivité ». RAXHON Philippe, "Essai de bilan historiographique de la mémoire ", Cahiers du Centre de recherches en histoire du droit et des Institutions, 2009, n`30, p. 15. 
de leur pouvoir symbolique dans le champ médiatique plus encore que de l'étendue de leur réseau de diffusion. Cette limite détermine alors un cadre pour évaluer concrètement les potentialités des forces de résistance et de transformation évoquées plus haut : pour qu'une "contre-mémoire " puisse faire l'objet d'une concurrence mémorielle médiatisée, il faut d'abord que son réseau de diffusion recoupe partiellement l'espace médiatique dominant, c'est-à-dire qu'elle puisse advenir, au moins potentiellement, sur la même scène médiatique.

Enfin, la question de la légitimation médiatique des mémoires apparaît comme une question éminemment politique, car elle renvoie inévitablement à celle des modalités de constitution de la communauté et du modèle de "vivre ensemble ". La médiatisation d'une mémoire revient en effet à attribuer cette mémoire à une communauté et, ce faisant, elle institue cette dernière de fait; c'est-à-dire qu'elle permet à la société d'identifier cette communauté en tant que communauté - y compris, d'ailleurs, pour les membres de cette communauté. Symétriquement, les silences médiatiques autour de certaines mémoires disqualifient les communautés porteuses de ces mémoires, qui ne sont dès lors pas reconnues en tant que communautés. Dans ce double geste performatif, les médias contribuent à dessiner les contours des communautés dont elles (dé)valorisent les mémoires, ainsi que les conditions de leur (dés)intégration en proposant un certain modèle de lien social.

\section{Reconfigiourations médiatiques et mémoires collectives : plu- ralisme oul émiettement ?}

La reconfiguration récente du paysage médiatique, liée au développement d'internet et à la prolifération corrélative des médias pose de nombreuses questions sur les modalités contemporaines de configuration de l'espace public et du collectif. En particulier, ces évolutions interrogent les formes médiatiques du consensus et de la concurrence mémorielles. Existe-t-il encore une scène commune pour le dialogue des mémoires, s'agit-il d'une pluralisation de la mémoire collective ou assiste-t-on au contraire à un émiettement mémoriel qui permettrait à une multiplicité de mémoires de coexister sans nécessairement se rencontrer?

En matière de mémoires, les "nouveaux médias " sont largement conçus comme une alternative aux médias de masse traditionnels, et comme étant capables de corriger certains défauts attribués à ces derniers. L'on pense surtout à cette tendance des médias dominants à reproduire des mémoires consensuelles, à cette difficulté à accueillir la conflictualité mémorielle, c'est-à-dire leur caractère plu- 
riel. S'inscrivant en faux contre l'uniformisation médiatique accusée de diluer les mémoires collectives en une mémoire globale, caractérisée par son homogénéité et la pauvreté de son contenu, les nouveaux modes de diffusion mémorielle sont le signe d'une réappropriation par les acteurs du discours médiatique en matière de mémoires et de l'émergence de contre-mémoires dans l'espace public. Ce phénomène est-il pour autant assimilable à un renouvellement des modes de légitimation des mémoires collectives ? Ce n'est pas tout à fait certain si l'on considère les remarques faites plus haut sur les rapports de force entre les réseaux de diffusion médiatique des mémoires.

Si en outre, comme nous en avons fait l'hypothèse, les frontières des espaces mémoriels recouvrent celles des différents espaces médiatiques - que ce soit au niveau de la production, de la diffusion ou de la réception - on peut se demander dans quelle mesure on n'assiste pas à un évidement relatif de la mémoire collective au profit d'une multitude de particularismes mémoriels, synonyme de phénomènes de replis mémoriels communautaires et d'une désarticulation des mémoires collectives.

Pris entre deux reproches, ou deux écueils, d'uniformatisation mémorielle d'une part et d'éparpillement mémoriel d'autre part - qui constituent au fond deux formes de l'oubli et de la méconnaissance pourrait-on imaginer quelque chose comme une "voie moyenne " pour les médias? En d'autres termes, à quelles conditions peut-on concevoir un modèle de médiatisation mémorielle qui permette d'intégrer la pluralité mémorielle en leur ménageant un espace commun, c'està-dire en admettant la possibilité de jouer la concurrence mémorielle, sans pour autant assimiler ces différentes voix dans une mémoire collective homogène et consensuelle?

\section{Entre consensus et concurrence mémorielles : quel modèle médiatique?}

On peut sans doute trouver un élément de réponse à cette question dans la distinction que fait Sébastien Fevry, dans le sillage des Memory Studies et des travaux d'Astrid Erll, entre «mémoire centripète » et «mémoire centrifuge ». Selon l'auteur, la première renverrait à une mémoire figée, "fermée sur elle-même, homogène et rétive à accueillir tout élément extérieur ". Cette forme de mémoire serait éminemment temporelle et territoriale, tout entière centrée sur le plaisir de la reconnaissance, de la répétition du même, et sur le désir de restauration de la communauté idéale perdue, ce qui explique son usage dans le cadre de politiques nationalistes. Dès lors, la mémoire de l'autre est nécessairement perçue comme une menace pour la 
pureté de la communauté. Dans ce modèle, il n'y a donc aucune place pour la concurrence mémorielle : une mémoire ne peut qu'être assimilée ou exclue.

La « mémoire centrifuge » en revanche, toujours d'après Sébastien Fevry, serait une mémoire "en mouvement", plus spatiale que territoriale, caractérisée par " un trafic constant entre différentes cultures et différentes époques ", ce qui l'identifie à une mémoire transculturelle. Plus réflexive, ce type de mémoire serait caractérisée par une force créatrice et une ouverture à l'altérité. Ainsi la mémoire centrifuge paraît-elle propre à accueillir, au moins potentiellement, la pluralité et la concurrence mémorielle tout en ménageant du commun.

Resterait alors à déterminer quel modèle médiatique pourrait véhiculer des mémoires de type centrifuge, de manière à articuler les mémoires particulières à la mémoire collective, à réaliser l'équilibre entre ce qui rassemble et ce qui sépare, entre les forces de reproduction et les forces de transformation. On pourrait certainement trouver une source d'inspiration dans les propositions de Géraldine Muhlmann, à la recherche d'un "idéal-critique » de journalisme qui puisse réaliser "la tension constitutive de la démocratie "6.

Sa question est au fond comparable à celle qui nous préoccupe: comment concevoir un modèle de journalisme qui permette à la fois de tisser du lien, du commun, et d'accueillir l'altérité, c'est-à-dire la possibilité du conflit? L'auteure propose à cette fin un modèle qu'elle baptise " journalisme du rassemblement conflictuel ", c'est-à-dire une forme médiatique qui œuvrerait à " décentrer jusqu'aux limites du lien » et qui rassemblerait dans cette épreuve même du décentrement. "Penser le journalisme à partir de cet idéal-critique, écrit-elle, c'est lui donner le rôle même de réaliser l'énigme démocratique, dans les conditions qui sont celles de notre modernité politique ${ }^{7}$.

Cette pensée de Géraldine Muhlmann fait par ailleurs écho à une réflexion générale sur les rapports entre médias et mémoire que nous aimerions partager au terme de ces quelques remarques conclusives. Nous avons tâché, dans ces quelques pages, de mettre en évidence quelques-unes des normes relatives aux relations entre médias et mémoires qui se dégagent de l'ensemble du numéro, et qui traversent chacune des recherches en dépit de la diversité de leurs approches ou de leurs objets. Or, l'horizon normatif de la médiatisation mémorielle qui se dessine au travers de ces études semble au fond intimement lié au questionnement démocratique lui-même, si bien que le « modèle »

${ }^{6}$ Munlmann Géraldine, Du journalisme en démocratie, Paris, Payot, 2004. 
médiatique que l'on pourrait concevoir en matière de mémoires reflète en fait l'idéal démocratique. Effectivement, la problématique du lien entre médias et mémoires engage inévitablement celle du lien social et des modalités de constitution du collectif. Dès lors, il n'est pas étonnant que le cadre normatif qui sous-tend les études sur la médiatisation de la mémoire soit traversé des mêmes tensions, des mêmes doutes, des mêmes ambiguïtés que la théorie démocratique : c'est que ces normes répondent à différentes conceptions de la démocratie et à différentes manières de concevoir la dialectique entre ouverture et clôture, consensus et conflit, censée constituer le socle du «vivre ensemble " de nos sociétés.

Jenifer DEVRESSE

Assistante au Département des Arts et Sciences de la Communication (LEMME) de l'Université de Liège

Pr. Dr Geoffrey GrandJEAN

Chargé de cours au Département de science politique de l'Université de Liège

Titulaire du cours « Mémoire et politique» 